\title{
Teledermatology: current indications and considerations for future use
}

\author{
Jacob Beer ${ }^{1} \cdot$ Edward Hadeler $^{2} \cdot$ Alejo Calume $^{3} \cdot$ Howard Gitlow $^{3} \cdot$ Keyvan Nouri $^{2}$ \\ Received: 16 September 2020 / Revised: 16 September 2020 / Accepted: 8 October 2020 / Published online: 19 October 2020 \\ (c) Springer-Verlag GmbH Germany, part of Springer Nature 2020
}

\begin{abstract}
Telemedicine is one of the most consequential technologies in modern healthcare. In certain situations, it allows for the delivery of care with high quality and minimal difficulty. This is particularly true in dermatology, in which many dermatological conditions can be treated remotely. The burden on dermatology patients has been greatly reduced for certain pathologies due to telemedicine. Health care providers also achieve improved job satisfaction following the convenience of meeting their patients. This paper details select dermatological conditions, and subsequently divides them into those treatable by telemedicine appointments, and those requiring face to face appointments.
\end{abstract}

Keywords Telemedicine $\cdot$ Teledermatology

\section{Introduction}

Telemedicine is among the recent pivotal advancements in modern medicine, allowing physicians to diagnose and administer treatments remotely, without having to be face to face with the patient. This practice involves electronic communication, where clinical services are provided without the patient having to visit the health care professional. The technology has been successfully incorporated into a number of clinical services: consultations, follow-ups visits, and medication prescriptions. Telemedicine has been observed to have ample benefits to both the health care provider and the patient, with one of the greatest being its improved access [1-4]. Perhaps the field most conducive to advances in telemedicine is dermatology, in which many diagnoses are made visually. While teledermatology has been widely incorporated, it does not allow for appropriate care in all skin conditions [5, 6]. Certain dermatologic conditions may

Jacob Beer

jacob.beer@pennmedicine.upenn.edu

1 Perelman School of Medicine, University of Pennsylvania, Philadelphia, PA, USA

2 Dr. Phillip Frost Department of Dermatology and Cutaneous Surgery, Miller School of Medicine, University of Miami, Miami, FL, USA

3 Miami Herbert Business School, University of Miami, Miami, FL, USA necessitate in-person visits, since not all treatments could be done through videos, images or a phone call.

\section{Methods}

This review was conducted by running keywords through PubMed and GoogleScholar. Search terms included teledermatology, telemedicine, store-and-forward, as well as cross-searches with the diseases of interest, including atopic dermatitis, acne, psoriasis, skin cancer, surveillance, melanoma, and pigmented lesions. Case reports, case series, systematic reviews, and original research articles spanning from January 2000 to August 2020 were thoroughly evaluated by 2 reviewers. Non-English studies were not included in this discussion.

\section{Literature review}

Ample research has been conducted on the use of telemedicine to study its effectiveness and its successful implementation [7]. Traditionally, patient outcomes, satisfaction and feasibility have been metrics to measure its effectiveness [8-10]. The two main mechanisms that exist in teledermatology are store-and-forward and synchronous platforms [11]. Store-and-forward refers to the practice of patients or primary care providers sending images of lesions of concern to dermatologists for evaluation. Synchronous 
teledermatology refers to the practice of live-stream virtual appointments between patients and dermatologists. The adequacies of each platform relies on the condition involved, the image quality, and the experience of the provider [8]. Data imply that doctors using store-and-forward appointments in teledermatology may attain more accurate results compared to face to face appointments in clinic because of the ease of second opinions [12]. Teledermatology has also been shown to improve access, and work as an effective triaging tool $[13,14]$.

Teledermatology has rapidly evolved in the United States ever, since it was introduced as a means of providing skincare to populations that could not access clinical centers [15]. A comprehensive study on teledermatology was conducted in the United States in 2011, which delineated many of the potential uses and drawbacks [16]. Programs were introduced to curb the rapidly growing demand for specialized skincare, and have continued to increase as more innovative measures are continuing to change the effectiveness of telemedicine on dermatology [16]. There are, however, several challenges facing teledermatology that could be a drawback to its success. Traditionally, one of the key barriers was the reimbursement visits relative to in-person visits [8]. However, in the era of COVID-19, this is less of a problem, as the Centers for Medicare and Medicaid Services have begun reimbursing virtual visits at the same rate as in-person visits [17]. Drawbacks to teledermatology still exist outside of reimbursement. Network connectivity remains an issue in remote areas or in those with poor internet connectivity. Similarly, socioeconomic status and technology literacy play roles in accessing teledermatology. Patients who do not have a smart phone, mobile device, or computer remain unable to have improved access. Communication between providers similarly remains problematic in certain cases [8]. While teledermatology has the potential to be used as an effective triaging tool, many referring practitioners are not well versed in referring patients through this communication [8]. Despite these drawbacks, teledermatology is largely considered successful at triaging and potentially managing many conditions. In general, these conditions can be considered based on the underlying etiology as well as the potential treatment used. In a recent study of the most common skin diseases in the United States, Lim et al. describe categories of prevalent diseases such as acne, atopic dermatitis, bullous diseases, cutaneous infections, connective tissue disorders, wounds, vitiligo, psoriasis and ulcers [18]. Of these, the conditions most heavily studied with respect to teledermatology have been atopic dermatitis, acne, psoriasis, and skin cancers. Specifically, the store-and-forward modality of teledermatology was evaluated in each of these conditions.

\section{Atopic dermatitis}

Telemedicine has been consistently shown to be effective in managing atopic dermatitis. As technology has improved, increasing studies have demonstrated the utility of store-and-forward teledermatology in atopic dermatitis. In 2015, researchers compared the effectiveness of telemedicine appointments to face to face appointments in a randomized clinical trial of 156 adults and children in hopes of understanding whether teledermatology could improve access to care for patients with atopic dermatitis [19]. Each patient attended a face to face appointment as the initial visit. Thereafter, half attended follow ups faceto-face with a dermatologist every 2 months for a year. The other half regularly attended their appointments by sending pictures of their skin to remote dermatologists that prescribed treatments remotely. The researchers measured the severity of eczema among all the patients at the initial office visit and each follow up visit using two standardized scoring systems. Results showed uniform improvement of the patients' atopic dermatitis across both groups. In 2017, a similarly designed study demonstrated that when atopic dermatitis patients were seen via telemedicine appointments, their quality of life outcomes improved to the same extent as those seen in person [20]. These studies support the inference that telemedicine appointments are as effective as face to face appointments for the treatment of atopic dermatitis.

\section{Acne}

Telemedicine has also been successful in effectively diagnosing and monitoring patients with acne. In a randomized controlled trial evaluating 69 patients diagnosed with severe acne that required isotretinoin therapy, patients received follow up care in the form of either outpatient face to face visits at 4-week intervals or telemedicine appointments every 2 weeks, each over a course of 24 weeks [21]. Telemedicine appointments utilized the store-and-forward modality, where patients captured three facial images and transmitted them over cellular networks to dermatologists for decision making. Using standardized scoring systems to assess treatment responsiveness, researchers found patients responded very positively to treatment regardless of whether the appointment was performed via telemedicine or as an in-person visit. In addition, patients who received the telemedicine modality reported high satisfaction with service throughout the study [21]. Further evidence of store-and-forward telemedicine as an effective diagnostic tool for acne was published in 2018 [22]. Researchers compared standardized scoring 
metrics of acne severity provided by dermatologists that used photos delivered remotely from patients (who were guided by an assistive phone application) to those provided by a dermatologist after an in-person examination. The study found assessment scores measuring disease severity agreed despite the change in modality, thereby demonstrating that telemedicine is as effective at diagnosing acne remotely.

\section{Psoriasis}

The treatment of psoriasis, which often requires extensive follow up, can also benefit from telemedicine. Early studies indicated the initial utility of teledermatology in the management of psoriasis, and these findings have been further validated by more recent findings. In 2010 , a study consisting of ten patients was conducted over a 12 -week period to identify whether store-and-forward teledermatology was as effective as face to face appointments in assessing the severity of psoriasis [23]. Teledermatology-treated patients used an assistive phone-based application to take pictures of areas of their affected skin. Two remote dermatologists then used those pictures to calculate the severity of each patient's disease at weeks $0,1,2$, and every 2 weeks thereafter for a total of 12 weeks. A third dermatologist calculated severity scores of the same patients in face to face appointments at weeks $0,1,6$, and 12 . Severity scores were very similar between the two methods. A study conducted 2 years later added evidence that psoriasis patients experience high patient satisfaction and improved quality of life when monitored remotely [24]. However, each study was limited by its relatively small sample size. Later studies augmented these early findings. In 2018, a randomized controlled trial evaluated 296 patients to determine whether standardized severity score indices varied between online store-and-forward or in-person treatments. Patients received either online or in-person care for psoriasis over a period of 12 months [25]. Each patient in the online group, sometimes in consultation with an in-person primary care physician, contacted dermatologists asynchronously and assessments and treatments were provided by the dermatologist online. In contrast, patients in the in-person group received treatment from dermatologists directly in face to face appointments. Results showed the store-and-forward online model was as effective as in-person management for psoriasis patients.

\section{Medication monitoring}

Telemedicine is effective in patients with three of the most common skin conditions diagnosed in dermatology: atopic dermatitis, acne, and psoriasis [18]. It has not only proven to be an effective diagnostic tool, but also has been shown to be effective for monitoring the treatment progression of these diseases. These findings encourage further exploration of the value of telemedicine in relation to diagnosing and treating other skin conditions, and emphasizes the value of store-and-forward teledermatology as a potential standard for medication follow up and monitoring of all dermatology patients.

\section{Skin cancer surveillance}

Despite its successful implementation in a series of dermatologic conditions, teledermatology has a more ambiguous use in melanocytic lesions, particularly with surveillance of skin cancers. With respect to patient satisfaction, teledermatology for use in skin cancer monitoring has been relatively successful [9]. This was found to be particularly true of younger patients, who have largely embraced teledermatology. In a recent literature review, Chuchu et al. note that in images of malignant lesions, the correct diagnosis was determined in the majority of cases [26]. However, they note several caveats, being the variable methodologies and types of malignancies among studies. Current recommendations detail that potentially malignant lesions require an in-person visit, citing a lack of systematic evidence detailing efficacy of teledermatology for this indication [27]. In recent studies, the use of teledermoscopy has been found to increase the accuracy of teledermatology with respect to diagnosing pigmented lesions [28]. This technology can be accessed through a smartphone or mobile device and highlights the potential for teledermatology with respect to surveillance for skin cancers. A relatively novel use is mole mapping, in which patients can take photographs of areas of concern to present to their dermatologist virtually [29]. While these methods have shown promise, many dermatologists are hesitant to adopt these technologies for monitoring potentially malignant processes, in part due to the lack of standardization across teledermatology practices as well as the legal implications.

\section{Discussion}

Telemedicine is observed to be gradually taking a central place in the delivery of healthcare. Conditions that require minimal attention and treatment can easily be treated through telemedicine as prescriptions are done online. However, telemedicine is limited in its use as it depends on a stable network connection to operate. A stable network connection is important in the process of communication between the patient and the health care professional, which is a key aspect of telemedicine. There needs to be constant communication to enable the health care professional to obtain direct and timely information about the condition and also to enable the patient to obtain accurate prescriptions 
in the right timings. Through such, telemedicine cannot be effective in areas that are lacking stable and secure internet connections, thereby limiting its use to a specific group of people.

In a bid to convert face to face appointments for healthcare to telemedicine appointments, there needs to be more advanced technologies in terms of the methods used in the provision of the network. A reliable and secure source supply of network should be established to ensure that the system is utilized effectively. Health care professionals can be urged to convert their services for outpatient visits to telemedicine. Face to face appointments can be done until full treatment can be accomplished through telemedicine appointments, by dermatological conditions. Virtual visits can also be encouraged for conditions that do not necessarily require close monitoring during treatment. Prescriptions of conditions that can be examined through pictures can be done online to minimize travel time. Doing so will also enable patients to cut on unnecessary costs associated with in-person visits. The software can be customized to enable a clear recording of patient information, which could easily be transferred to other health care professionals or other health care facilities when required. Doing so would help improve telemedicine services, thereby recognizing its advantages over face to face care.

Telemedicine can improve by ensuring that the privacy of its patients is considered. Privacy can be reached by ensuring that login details and a consent enquiry are required before any information is presented to the health care professional about the patient. Information about the patient can also be encrypted through the use of more customized software. Through the Health Insurance Portability and Accountability Act of 1996 (HIPAA), patients' medical records and confidential health information are able to be protected [30]. Appropriate navigation of HIPAA is crucial to the successful implementation of telemedicine. Electronic billing is used in keeping records thereby minimizing the chances of fraud in health care delivery. Devices can ensure the security of information using passwords. These ensure that no unauthorized persons can access confidential information about a patient's condition. As telemedicine continues to be a great contribution to health care across the world, it is necessary to conduct further research on innovations, its success, and various solutions to the shifting from in-person treatment to telemedicine.

\section{Conclusion}

While the COVID-19 pandemic has driven renewed interest in teledermatology, we anticipate improved access to healthcare and overall satisfaction from patients and providers will continue to propel its growth. Many studies have verified teledermatology as a valid treatment for select chronic skin conditions such as atopic dermatitis, acne, and psoriasis. However, the practice still has opportunities to expand, including in the diagnosis, surveillance and treatment of skin cancer, where new technologies are being explored to provide remote monitoring capabilities for suspicious skin lesions.

Funding None.

\section{Compliance with ethical standards}

Conflict of interest The author(s) declare that they have no conflicts of interest.

\section{References}

1. Bystryn JC (2000) Dermatology manpower needs. DermatolClin 18(2):303-312

2. Resneck J Jr, Kimball AB (2004) The dermatology workforce shortage. J Am AcadDermatol 50(1):50-54

3. Suneja T, Smith ED, Chen GJ, Zipperstein KJ, Fleischer AB Jr, Feldman SR (2001) Waiting times to see a dermatologist are perceived as too long by dermatologists: implications for the dermatology workforce. Arch Dermatol 137(10):1303-1307

4. Tsang MW, Resneck JS Jr (2006) Even patients with changing moles face long dermatology appointment wait-times: a study of simulated patient calls to dermatologists. J Am AcadDermatol 55(1):54-58

5. Warshaw EM, Lederle FA, Grill JP et al (2009) Accuracy of teledermatology for pigmented neoplasms. J Am AcadDermatol 61(5):753-765

6. Tandjung R, Badertscher N, Kleiner N et al (2015) Feasibility and diagnostic accuracy of teledermatology in Swiss primary care: process analysis of a randomized controlled trial. J EvalClinPract 21(2):326-331

7. Hartvigsen G, Johansen MA, Hasvold P et al (2007) Challenges in telemedicine and eHealth: lessons learned from 20 years with telemedicine in Tromso. Stud Health Technol Inform 129(Pt 1):82-86

8. Lee KJ, Finnane A, Soyer HP (2018) Recent trends in teledermatology and teledermoscopy. DermatolPract Concept $8(3): 214-223$

9. Nicholson P, Macedo C, Fuller C, Thomas L (2020) Patient satisfaction with a new skin cancer teledermatology service. ClinExpDermatol 45(6):691-698

10. Hadeler E, Gitlow H, Nouri K (2020) Definitions, survey methods, and findings of patient satisfaction studies in teledermatology: a systematic review. Arch Dermatol Res

11. Pasquali P, Sonthalia S, Moreno-Ramirez D et al (2020) Teledermatology and its current perspective. Indian Dermatol Online J 11(1):12-20

12. Lozzi GP, Soyer HP, Massone $C$ et al (2007) The additive value of second opinion teleconsulting in the management of patients with challenging inflammatory, neoplastic skin diseases: a best practice model in dermatology? J EurAcadDermatolVenereol 21(1):30-34

13. Borve A, DahlenGyllencreutz J, Terstappen K et al (2015) Smartphone teledermoscopy referrals: a novel process for improved triage of skin cancer patients. Acta DermVenereol 95(2):186-190 
14. Uscher-Pines L, Malsberger R, Burgette L, Mulcahy A, Mehrotra A (2016) Effect of teledermatology on access to dermatology care among Medicaid enrollees. JAMA Dermatol 152(8):905-912

15. Yim KM, Florek AG, Oh DH, McKoy K, Armstrong AW (2018) Teledermatology in the United States: an update in a dynamic era. Telemed J E Health 24(9):691-697

16. Armstrong AW, Wu J, Kovarik CL et al (2012) State of teledermatology programs in the United States. J Am AcadDermatol 67(5):939-944

17. Beer J, Abrouk M, Kirsner R (2020) Telemedicine platforms used in academic dermatology during the COVID-19 pandemic: implications for adaptation and usage. J Drugs Dermatol 19(8):797-798

18. Lim HW, Collins SAB, Resneck JS Jr et al (2017) The burden of skin disease in the United States. J Am AcadDermatol 76(5):958972.e952

19. Armstrong AW, Johnson MA, Lin S, Maverakis E, Fazel N, Liu FT (2015) Patient-centered, direct-access online care for management of atopic dermatitis: a randomized clinical trial. JAMA Dermatol 151(2):154-160

20. Kornmehl H, Singh S, Johnson MA, Armstrong AW (2017) Direct-access online care for the management of atopic dermatitis: a randomized clinical trial examining patient quality of life. Telemed J E Health 23(9):726-732

21. Fruhauf J, Krock S, Quehenberger F et al (2015) Mobile teledermatology helping patients control high-need acne: a randomized controlled trial. J EurAcadDermatolVenereol 29(5):919-924

22. Singer HM, Almazan T, Craft N et al (2018) Using network oriented research assistant (NORA) technology to compare digital photographic with in-person assessment of acne vulgaris. JAMA Dermatol 154(2):188-190

23. Fruhauf J, Schwantzer G, Ambros-Rudolph CM et al (2010) Pilot study using teledermatology to manage high-need patients with psoriasis. Arch Dermatol 146(2):200-201
24. Fruhauf J, Schwantzer G, Ambros-Rudolph CM et al (2012) Pilot study on the acceptance of mobile teledermatology for the home monitoring of high-need patients with psoriasis. Australas J Dermatol 53(1):41-46

25. Armstrong AW, Chambers CJ, Maverakis E et al (2018) Effectiveness of online vs in-person care for adults with psoriasis: a randomized clinical trial. JAMA Netw Open 1(6):e183062

26. Chuchu N, Dinnes J, Takwoingi Y et al (2018) Teledermatology for diagnosing skin cancer in adults. Cochrane Database Syst Rev 12:CD013193

27. In: 2019 surveillance of melanoma: assessment and management (NICE guideline NG14) and improving outcomes for people with skin tumours including melanoma (NICE guideline CSG8). London2019

28. Tognetti L, Cartocci A, Balistreri A, et al (2020) The comparative use of multiple electronic devices in the teledermoscopic diagnosis of early melanoma. Telemed J E Health

29. Pala P, Bergler-Czop BS, Gwizdz JM (2020) Teledermatology: idea, benefits and risks of modern age - a systematic review based on melanoma. PostepyDermatolAlergol 37(2):159-167

30. Luxton DD, Kayl RA, Mishkind MC (2012) mHealth data security: the need for HIPAA-compliant standardization. Telemed J E Health 18(4):284-288

Publisher's Note Springer Nature remains neutral with regard to jurisdictional claims in published maps and institutional affiliations. 